\title{
On the Discretization in Time of Semilinear Parabolic Equations with Nonsmooth Initial Data
}

\author{
By Michel Crouzeix and Vidar Thomée
}

\begin{abstract}
Single-step discretization methods are considered for equations of the form $u_{t}+A u$ $=f(t, u)$, where $A$ is a linear positive definite operator in a Hilbert space $H$. It is shown that if the method is consistent with the differential equation then the convergence is essentially of first order in the stepsize, even if the initial data $v$ are only in $H$, but also that, in contrast to the situation in the linear homogeneous case, higher-order convergence is not possible in general without further assumptions on $v$.
\end{abstract}

1. Introduction. We shall begin by recalling some results concerning the discretization in time of the linear homogeneous equation

$$
\begin{aligned}
& u_{t}+A u=0 \text { for } t>0, u_{t}=\partial u / \partial t, \\
& u(0)=v,
\end{aligned}
$$

where $A$ is a selfadjoint positive definite operator in a Hilbert space $H$ (cf., e.g., Baker, Bramble and Thomée [1]).

Let $r(z)$ be a rational function having no poles for $z \geqslant 0$, and define an approximate solution $U_{n}$ at $t=t_{n}=n k$, where $k$ is the time step, by

$$
\begin{aligned}
& U_{n+1}=r(k A) U_{n} \text { for } n=0,1,2, \ldots, \\
& U_{0}=v .
\end{aligned}
$$

Assume that the approximation is of order $p$ with $p \geqslant 1$, or

$$
r(z)=e^{-z}+O\left(z^{p+1}\right) \quad \text { as } z \rightarrow 0,
$$

and also that the method is stable in the sense that

$$
|r(z)| \leqslant 1 \text { for } z \geqslant 0 \text {. }
$$

Then one may show the "smooth data" error estimate

$$
\left\|U_{n}-u\left(t_{n}\right)\right\| \leqslant C k^{p}\left\|A^{p} v\right\| \text { for } v \in D\left(A^{p}\right) .
$$

This follows easily from spectral representations and the fact that under our assumptions

$$
\left|r(z)^{n}-e^{-n z}\right| \leqslant C z^{p} \text { for } z \geqslant 0 .
$$

In applications, the requirement $v \in D\left(A^{p}\right)$ is quite restrictive. For example, if $A$ is associated with an elliptic partial differential operator in a domain $\Omega \subset R^{d}$, it demands not only smoothness of the initial data, but also that they satisfy certain

Received April 1, 1985.

1980 Mathematics Subject Classification (1985 Revision). Primary 65M15.

(C1987 American Mathematical Society $0025-5718 / 87 \$ 1.00+\$ .25$ per page 
compatibility conditions at the boundary $\partial \Omega$ for $t=0$. However, under the stronger stability assumption

$$
|r(z)|<1 \text { for } z>0, \text { and }|r(\infty)|<1,
$$

one can also show the "nonsmooth" data error estimate

$$
\left\|U_{n}-u\left(t_{n}\right)\right\| \leqslant C k^{p} t_{n}^{-p}\|v\| \text { for } v \in H, t_{n}>0 .
$$

This follows again by spectral arguments from

$$
\left|r(z)^{n}-e^{-n z}\right| \leqslant C n^{-p} \quad \text { for } z \geqslant 0,
$$

and shows that even with $v$ only in $H$, the $O\left(k^{p}\right)$ convergence is retained for $t_{n}>0$. It follows also that for $0 \leqslant q \leqslant p$ the intermediate estimates

$$
\left\|U_{n}-u\left(t_{n}\right)\right\| \leqslant C k^{p} t_{n}^{-q}\left\|A^{p-q} v\right\| \text { for } v \in D\left(A^{p-q}\right)
$$

hold.

The question we want to address below is to what extent these error estimates with reduced regularity assumptions carry over to semilinear equations. Thus assume that $f(t, u)$ is a smooth function on $\bar{J} \times H$, where $J=(0, T]$ with $T<\infty$, and consider the semilinear problem

$$
\begin{aligned}
& u_{t}+A u=f(t, u) \quad \text { for } t \in J, \\
& u(0)=v .
\end{aligned}
$$

For its approximate solution we will investigate in Section 2 single-step discretization schemes of the form

$$
\begin{aligned}
& U_{n+1}=r(k A) U_{n}+k F\left(k, t_{n}, U_{n}\right) \text { for } t_{n} \in \bar{J}, \\
& U_{0}=v,
\end{aligned}
$$

where $r(z)$ satisfies (1.2) with $p=1$ and $F(k, t, v)$ is chosen to be consistent with (1.6) in a sense to be made precise below. As an example of such schemes, consider the standard first-order backward Euler scheme defined by

$$
U_{n+1}=(I+k A)^{-1} U_{n}+k(I+k A)^{-1} f\left(t_{n+1}, U_{n+1}\right),
$$

or the linearized version

$$
U_{n+1}=(I+k A)^{-1} U_{n}+k(I+k A)^{-1} f\left(t_{n}, U_{n}\right),
$$

where in the first case $F\left(k, t_{n}, U_{n}\right)$ is defined implicitly by (1.7). We shall be able to show (Theorem 1) that for such schemes

$$
\left\|U_{n}-u\left(t_{n}\right)\right\| \leqslant C k t_{n}^{-1} \log \frac{t_{n+1}}{k} \text { for } t_{n} \in J
$$

where $C$ depends on an upper bound for $\|v\|$, so that for first-order schemes the estimate (1.4) essentially remains valid in the semilinear case.

In Section 3 we briefly recall the definitions and basic properties of Runge-Kutta methods (cf., e.g., Crouzeix [2]) and show that our result above applies to such methods.

In Section 4 we shall then demonstrate that, more surprisingly, it is not in general possible to generalize the higher-order estimate (1.4) with $p>1$ to semilinear equations. This will be done by exhibiting a simple system of the form (1.6) such 
that, for any choice of a Runge-Kutta method satisfying (1.3), and any $t \in J$, we have

$$
\limsup _{n=t / k \rightarrow \infty}\left\|U_{n}-u\left(t_{n}\right)\right\| \geqslant c k \quad \text { with } c=c(t)>0 .
$$

We shall then proceed, in Section 5, within the framework of Runge-Kutta methods satisfying (1.3), to show (Theorem 2) that if the method is accurate of order $p$, with order $p-1$ for the intermediate equations (cf. Section 5), then, if $u^{(j)}(t)$ are bounded for $j<p$ together with $t u^{(p)}(t), f(t, u(t))^{(j)}, j<p$, and $t f(t, u(t))^{(p)}$, we have

$$
\left\|U_{n}-u\left(t_{n}\right)\right\| \leqslant C k^{p}\left(t_{n}^{-1} \log \frac{t_{n+1}}{k}+\left(\log \frac{t_{n+1}}{k}\right)^{2}\right) \text { for } t_{n} \in J,
$$

which is thus an analogue of (1.5) with $q=1$. Again, in practice, these assumptions will require certain compatibility conditions at $t=0$.

These investigations are in a sense a continuation of work by Johnson, Larsson, Thomée and Wahlbin [3] concerning finite element type discretization with respect to the space variables of semilinear parabolic equations, and as we shall see below, our present results may be combined with those of [3] to yield error bounds for completely discrete schemes. The fact that the nonsmooth data error estimates for the linear homogeneous equation do not generalize to the semilinear problem for higher-order methods was shown in the case of semidiscretization in space in [3] by a counterexample, which was the starting point of this work.

2. The First-Order Error Estimate for Nonsmooth Data. Consider the initial value problem

$$
\begin{aligned}
& u_{t}+A u=f(t, u) \text { for } t \in J=(0, T] \\
& u(0)=v,
\end{aligned}
$$

where $A$ is a selfadjoint positive definite operator in a Hilbert space $H$ and where $f(t, u)$ has values in $H$ and is continuous and bounded together with its first-order derivatives with respect to $t$ and $u$ for $(t, u) \in \bar{J} \times H$. This problem has a unique solution on $J$ for $v \in H$, which satisfies the integral equation

$$
u(t)=E(t) v+\int_{0}^{t} E(t-s) f(s, u(s)) d s,
$$

where $E(t)$ is the semigroup generated by $-A$. This semigroup is analytic, since by spectral representation

$$
t\|A E(t) v\| \leqslant \sup _{z \geqslant 0}\left(t z e^{-t z}\right)\|v\|=C\|v\|,
$$

so that, in particular, for the solution of the homogeneous linear problem (1.1),

$$
\left\|u_{t}(t)\right\|=\|A u(t)\|=\|A E(t) v\| \leqslant \frac{C}{t}\|v\| \quad \text { for } t \in J
$$

and for the solution of (1.1) we also have that $u_{t}$ is bounded in $H$ if $v \in D(A)$, i.e.,

$$
\left\|u_{t}(t)\right\|=\|E(t) A v\| \leqslant\|A v\| .
$$


We shall need the corresponding results for the solution of our semilinear problem (2.1).

LEMMA 1. There are constants $C_{i}=C_{i}(\rho), i=0,1$, such that the solution of (2.1) satisfies

$$
\left\|u_{t}(t)\right\| \leqslant C_{0} t^{-1} \quad \text { for all } v \in H \text { with }\|v\| \leqslant \rho
$$

and

$$
\left\|u_{t}(t)\right\| \leqslant C_{1} \quad \text { for all } v \in D(A) \text { with }\|A v\| \leqslant \rho .
$$

The constants $C_{i}, i=0,1$, depend only on $\rho$ and on bounds in $H$ for $f, f_{t}$ and $f_{u}$ and are independent of the Hilbert space $H$ and the positive definite operator $A$.

Proof. We introduce the symmetric, positive definite bilinear form

$$
a(v, w)=(A v, w) \text { for } v, w \in D(A),
$$

which we may consider extended to the subspace $V$ of $H$ defined by the norm $\|v\|_{V}=a(v, v)^{1 / 2}$. We may then write our differential equation in weak form

$$
\left(u_{t}, \varphi\right)+a(u, \varphi)=(f(t, u), \varphi) \text { for } \varphi \in V .
$$

We obtain by differentiation with respect to $t$, which is legitimate since the equation obtained is linear in $u_{t}$, with bounded coefficients,

$$
\left(u_{t t}, \varphi\right)+a\left(u_{t}, \varphi\right)=\left(f_{t}(t, u)+f_{u}(t, u)\left(u_{t}\right), \varphi\right) \text { for } t \in J,
$$

and hence with $\varphi=u_{t}$,

$$
\frac{1}{2} \frac{d}{d t}\left\|u_{t}\right\|^{2}+\left\|u_{t}\right\|_{V}^{2} \leqslant C\left(\left\|u_{t}\right\|+1\right)\left\|u_{t}\right\| .
$$

This yields

$$
\frac{d}{d t}\left\|u_{t}\right\| \leqslant C\left\|u_{t}\right\|+C
$$

and, since $J$ is bounded,

$$
\left\|u_{t}(t)\right\| \leqslant C\left\|u_{t}(0)\right\|+C \leqslant C\|A v\|+C \leqslant C_{1}(\rho),
$$

which is the second statement of the lemma.

In order to show the first, we choose $\varphi=t^{2} u_{t}$ in (2.3) to obtain

$$
\frac{1}{2} \frac{d}{d t}\left(t^{2}\left\|u_{t}\right\|^{2}\right)+t^{2}\left\|u_{t}\right\|_{V}^{2}=t^{2}\left(f_{t}+f_{u}\left(u_{t}\right), u_{t}\right)+t\left\|u_{t}\right\|^{2} \leqslant C t\left\|u_{t}\right\|^{2}+C,
$$

and thus

$$
t^{2}\left\|u_{t}(t)\right\|^{2} \leqslant C \int_{0}^{t} s\left\|u_{t}\right\|^{2} d s+C .
$$

Taking $\varphi=t u_{t}$ in (2.2), we find

$$
t\left\|u_{t}\right\|^{2}+\frac{1}{2} \frac{d}{d t}\left(t\|u\|_{V}^{2}\right)=t\left(f(t, u), u_{t}\right)+\frac{1}{2}\|u\|_{V}^{2}
$$

and hence

$$
\int_{0}^{t} s\left\|u_{t}(s)\right\|^{2} d s \leqslant C \int_{0}^{t}\|u\|_{v}^{2} d s+C
$$


Finally, $\varphi=u$ in (2.2) gives similarly

$$
\int_{0}^{t}\|u\|_{v}^{2} d s \leqslant\|v\|^{2}+C \leqslant C_{0}(\rho)
$$

which completes the proof.

As in the introduction, we consider now a difference scheme for (2.1) of the form

$$
\begin{aligned}
& U_{n+1}=E_{k} U_{n}+k F\left(k, t_{n}, U_{n}\right) \text { for } t_{n} \in \bar{J}, \\
& U_{0}=v,
\end{aligned}
$$

where $E_{k}=r(k A)$ for some rational function $r$ satisfying (1.2) with $p=1$ and (1.3), and where $F(k, t, \varphi)$ is a sufficiently smooth function chosen to make (2.4) consistent with (2.1). For a finite-dimensional problem this would simply mean that

$$
F(0, t, \varphi)=f(t, \varphi)
$$

in the general Hilbert space context we shall need to make this more precise. We shall thus assume that $F$ is uniformly Lipschitz continuous will respect to $\varphi$, so that, for some $k_{0}>0$,

$$
\|F(k, t, \varphi)-F(k, t, \psi)\| \leqslant C\|\varphi-\psi\| \text { on }\left[0, k_{0}\right] \times \bar{J} \times H,
$$

and, in addition, that

$$
\left\|A^{-1}(F(k, t, \varphi)-f(t, \varphi))\right\| \leqslant C k(\|A \varphi\|+1) \quad \text { on }\left[0, k_{0}\right] \times \bar{J} \times D(A) .
$$

Note that the latter condition follows from (2.5) in the finite-dimensional case if $F$ is Lipschitz continuous with respect to $k$. Observe also that (2.6) implies

$$
\|F(k, t, \varphi)\| \leqslant C(1+\|\varphi\|) \text { on }\left[0, k_{0}\right] \times \bar{J} \times H .
$$

We are now ready to state and prove the main result of this section.

THEOREM 1. Assume for the difference scheme (2.4) that $E_{k}=r(k A)$, where $r$ satisfies (1.2) with $p=1$ and (1.3), and that (2.6) and (2.7) hold. Then there is a constant $C=C(\rho)$ such that for $t_{n} \in J$

$$
\left\|U_{n}-u\left(t_{n}\right)\right\| \leqslant C\left(\frac{k}{t_{n}} \log \frac{t_{n+1}}{k}+k\left(\log \frac{t_{n+1}}{k}\right)^{2}\right) \quad \text { for } v \in H \text { with }\|v\| \leqslant \rho .
$$

The constant $C$ depends, in addition to $\rho$, only on bounds for $f, f_{t}, f_{u}$ and on the constants of (2.6) and (2.7) and is independent of the particular choice of the Hilbert space $H$ and the positive definite operator $A$.

Proof. We find at once

$$
U_{n}=E_{k}^{n} v+k \sum_{j=0}^{n-1} E_{k}^{n-1-j} F\left(k, t_{j}, U_{j}\right) .
$$

For the exact solution we may write similarly, with $I_{j}=\left(t_{j}, t_{j+1}\right)$ and $u_{n}=u\left(t_{n}\right)$,

$$
u_{n}=E\left(t_{n}\right) v+\sum_{j=0}^{n-1} \int_{I_{j}} E\left(t_{n}-s\right) f(s, u(s)) d s
$$

so that for the error, $e_{n}=U_{n}-u_{n}$,

$$
e_{n}=\left(E_{k}^{n}-E\left(t_{n}\right)\right) v+\sum_{j=0}^{n-1} \int_{I_{j}} D_{n, j}(s) d s
$$


where

$$
D_{n, j}(s)=E_{k}^{n-1-j} F\left(k, t_{j}, U_{j}\right)-E\left(t_{n}-s\right) f(s, u(s)) .
$$

We write this latter expression in the form

$$
\begin{aligned}
D_{n, j}(s)= & E_{k}^{n-1-j}\left(F\left(k, t_{j}, U_{j}\right)-F\left(k, t_{j}, u_{j}\right)\right) \\
& +\left(E_{k}^{n-1-j}-E\left(t_{n-1-j}\right)\right) F\left(k, t_{j}, u_{j}\right) \\
& +E\left(t_{n-1-j}\right)\left(F\left(k, t_{j}, u_{j}\right)-f\left(t_{j}, u_{j}\right)\right) \\
& +E\left(t_{n-1-j}\right)\left(f\left(t_{j}, u_{j}\right)-f(s, u(s))\right. \\
& +\left(E\left(t_{n-1-j}\right)-E\left(t_{n}-s\right)\right) f(s, u(s)) \\
= & \sum_{l=1}^{5} d_{j, l}(s) .
\end{aligned}
$$

We now proceed to estimate these five terms for $s \in I_{j}$. We first have, by the stability of $E_{k}$ and (2.6),

$$
\left\|d_{j, 1}\right\| \leqslant C\left\|U_{j}-u_{j}\right\|=C\left\|e_{j}\right\| .
$$

For the second term we note that (1.4) may be written

$$
\left\|\left(E_{k}^{n}-E\left(t_{n}\right)\right) v\right\| \leqslant C \frac{k}{t_{n}}\|v\| \text { for } t_{n}>0,
$$

and we conclude, by (2.8),

$$
\left\|d_{j, 2}\right\| \leqslant C \frac{k}{t_{n-1-j}} \text { for } j \neq n-1 .
$$

Since $d_{n-1,2}=0$ we may write

$$
\left\|d_{j, 2}\right\| \leqslant \frac{C k}{t_{n-j}} \text { for } 0 \leqslant j \leqslant n-1 .
$$

For the third term we use the consistency condition (2.7), the analyticity of $E(t)$ and Lemma 1 to obtain for $0<j<n-1$,

$$
\begin{aligned}
\left\|d_{j, 3}\right\| & =\left\|A E\left(t_{n-1-j}\right) A^{-1}\left(F\left(k, t_{j}, u_{j}\right)-f\left(t_{j}, u_{j}\right)\right)\right\| \\
& \leqslant \frac{C}{t_{n-1-j}} k\left(\left\|A u_{j}\right\|+1\right) \leqslant C \frac{k}{t_{n-1-j}}\left(\left\|u_{t}\left(t_{j}\right)\right\|+1\right) \leqslant C \frac{k}{t_{j} t_{n-1-j}} .
\end{aligned}
$$

For $j=0$ and $n-1$ we find easily by the boundedness of $F$ and $f$,

$$
\left\|d_{j, 3}\right\| \leqslant C=C \frac{k}{t_{1}} \leqslant C \frac{k}{t_{1} t_{n}}
$$

so that we may write

$$
\left\|d_{j, 3}\right\| \leqslant C \frac{k}{t_{j+1} t_{n-j}}=\frac{C k}{t_{n}}\left(\frac{1}{t_{j+1}}+\frac{1}{t_{n-j}}\right), \quad 0 \leqslant j \leqslant n-1 .
$$

For the fourth term we have

$$
\begin{aligned}
\left\|d_{j, 4}\right\| & \leqslant\left\|f\left(t_{j}, u_{j}\right)-f(s, u(s))\right\| \leqslant C\left(k+\left\|u_{j}-u(s)\right\|\right) \\
& \leqslant C k\left(\sup _{I_{j}}\left\|u_{t}(s)\right\|+1\right) \leqslant C \frac{k}{t_{j}} \quad \text { for } 0<j \leqslant n-1,
\end{aligned}
$$


and, since $d_{0,4}$ is bounded,

$$
\left\|d_{j, 4}\right\| \leqslant C \frac{k}{t_{j+1}} \quad \text { for } 0 \leqslant j \leqslant n-1
$$

Finally,

$$
\begin{aligned}
\left\|d_{j, 5}\right\| & =\left\|A E\left(t_{n-1-j}\right) A^{-1}\left(E\left(t_{j+1}-s\right)-I\right) f(s, u(s))\right\| \\
& \leqslant C \frac{k}{t_{n-1-j}} \quad \text { for } j<n-1,
\end{aligned}
$$

where we have used the analyticity of $E(t)$ and the fact that

$$
\left\|A^{-1}\left(E\left(t_{j+1}-s\right)-I\right) v\right\| \leqslant \sup _{z>0}\left|\frac{e^{-\left(t_{j+1}-s\right) z}}{z}\right| \cdot\|v\| \leqslant C k\|v\| .
$$

Again,

$$
\left\|d_{n-1,5}\right\| \leqslant C=C \frac{k}{t_{1}}
$$

so that

$$
\left\|d_{j, 5}\right\| \leqslant C \frac{k}{t_{n-j}} \text { for } 0 \leqslant j \leqslant n-1 .
$$

Altogether we have thus from (2.9), using (2.10) to estimate the first term, for $\|v\| \leqslant \rho$,

$$
\left\|e_{n}\right\| \leqslant C \frac{k}{t_{n}}+C k \sum_{j=0}^{n-1}\left\|e_{j}\right\|+C \frac{k}{t_{n}} \sum_{j=1}^{n} \frac{k}{t_{j}} \leqslant C k \sum_{j=0}^{n-1}\left\|e_{j}\right\|+C \frac{k}{t_{n}} \log (n+1) ;
$$

setting $\beta_{n}=k \sum_{j=0}^{n}\left\|e_{j}\right\|$, we thus have

$$
\beta_{n} \leqslant(1+C k) \beta_{n-1}+\frac{k}{n} \log (n+1),
$$

and hence

$\beta_{n} \leqslant k \sum_{j=1}^{n}(1+C k)^{n-1-j} \frac{1}{j} \log (j+1) \leqslant C k \log (n+1) \sum_{j=1}^{n} \frac{1}{j} \leqslant C k(\log (n+1))^{2}$

and finally

$$
\begin{aligned}
\left\|e_{n}\right\| & \leqslant C k(\log (n+1))^{2}+C \frac{k}{t_{n}} \log (n+1) \\
& =C\left(\frac{k}{t_{n}} \log \frac{t_{n+1}}{k}+k\left(\log \frac{t_{n+1}}{k}\right)^{2}\right),
\end{aligned}
$$

which completes the proof of the theorem.

As our first example we consider the linearized modification of the backward Euler scheme defined in (1.8). Here,

$$
F(k, t, \varphi)=(I+k A)^{-1} f(t, \varphi),
$$


and it is clear from our assumption on $f$ that (2.6) is satisfied. Further, we note that

and hence

$$
\begin{aligned}
A^{-1}(F(k, t, \varphi)-f(t, \varphi)) & =k(k A)^{-1}\left((I+k A)^{-1}-I\right) f(t, \varphi) \\
& =k(I+k A)^{-1} f(t, \varphi)
\end{aligned}
$$

$$
\left\|A^{-1}(F(k, t, \varphi)-f(t, \varphi))\right\| \leqslant C k,
$$

so that (2.7) holds even without the term $\|A \varphi\|$. For schemes with this property the result of Theorem 1 is in fact valid without the term $C k\left(\log \left(t_{n+1} / k\right)\right)^{2}$.

Turning now to the standard backward Euler scheme, we have here

$$
F(k, t, \varphi)=(I+k A)^{-1} f(t+k, \Psi),
$$

where $\Psi=\Psi(\varphi)$ is obtained from the nonlinear equation

$$
\Psi=(I+k A)^{-1}(\varphi+k f(t+k, \Psi)) .
$$

It is clear by the contraction mapping theorem that this equation has a unique solution $\Psi$ for small $k$ and that $\Psi$ depends Lipschitz continuously on $\varphi$. Obviously, again (2.6) is satisfied for this scheme. As for (2.7), we have

and thus

$$
\begin{aligned}
\Psi-\varphi & =\Psi-(I+k A)^{-1} \varphi-(I+k A)^{-1} k A \varphi \\
& =k(I+k A)^{-1} f(t+k, \Psi)-(I+k A)^{-1} k A \varphi,
\end{aligned}
$$

Further,

$$
\|\Psi-\varphi\| \leqslant C k(1+\|A \varphi\|) .
$$

$$
\begin{aligned}
F(k, t, \varphi)-f(t, \varphi)= & (I+k A)^{-1}(f(t+k, \Psi)-f(t+k, \varphi)) \\
& +(I+k A)^{-1}(f(t+k, \varphi)-f(t, \varphi)) \\
& -(I+k A)^{-1} k A f(t, \varphi)
\end{aligned}
$$

and hence, since $A^{-1}$ is bounded,

$$
\left\|A^{-1}(F(k, t, \varphi)-f(t, \varphi))\right\| \leqslant C\|\Psi-\varphi\|+C k \leqslant C k(1+\|A \varphi\|),
$$

which is the desired estimate.

The above result may be applied to parabolic problems which have already been discretized in the space variables. For instance, for concreteness, consider the case that $H=L_{2}(\Omega)$, where $\Omega$ is a domain with smooth boundary in $R^{d}$, where $A=-\Delta$, with $D(A)=H_{0}^{1}(\Omega) \cap H^{2}(\Omega)$, and where $f(t, u)$ is generated by a smooth function $\tilde{f}(x, t, u)$ on $\bar{\Omega} \times \bar{J} \times R$ which is bounded together with its first-order derivatives with respect to $t$ and $u$. Now let $S_{h} \subset H_{0}^{1}(\Omega)$ consist of continuous, piecewise linear functions on a partitioning of $\Omega$ into simplices and let $u_{h}: \bar{J} \rightarrow S_{h}$ be defined by

$$
\begin{aligned}
& \left(u_{h, t}, \chi\right)+\left(\nabla u_{h}, \nabla \chi\right)=\left(\tilde{f}\left(\cdot, t, u_{h}\right), \chi\right) \text { for } \chi \in S_{h}, t \in J, \\
& u_{h}(0)=v_{h},
\end{aligned}
$$

where $(\cdot, \cdot)$ denotes the standard inner products on $L_{2}(\Omega)$ and $L_{2}(\Omega)^{d}$. Defining the discrete solution operator $T_{h}: L_{2}(\Omega) \rightarrow S_{h}$ of the associated elliptic problem by

$$
\left(\nabla T_{h} w, \nabla \chi\right)=(w, \chi) \text { for } \chi \in S_{h},
$$

and setting $\Delta_{h}=-T_{h}^{-1},(2.11)$ may be written in the form (2.1) as

$$
u_{h, t}-\Delta_{h} u_{h}=P_{0} \tilde{f}\left(\cdot, t, u_{h}\right) \quad \text { for } t \in J
$$


where $P_{0}$ denotes the orthogonal $L_{2}$-projection onto $S_{h}$. From [3] it is known that, if $v_{h}=P_{0} v$, the error in this space discretization satisfies

$$
\left\|u_{h}(t)-u(t)\right\| \leqslant C(\rho) \frac{h^{2}}{t} \log \frac{t}{h^{2}} \quad \text { for all } v \in L_{2}(\Omega) \text { with }\|v\| \leqslant \rho,
$$

where $h$ is the maximal diameter of the simplices of the partitioning.

Our above analysis applies to this situation and yields estimates which are uniform in $h$. In fact, if $\tilde{f}$ is as above, then $P_{0} \tilde{f}(x, t, u)$ generates a function $\bar{J} \times S_{h} \ni\left(t, u_{h}\right) \rightarrow P_{0} \tilde{f}\left(\cdot, t, u_{h}\right) \in S_{h}$ which satisfies our above assumptions with respect to the Hilbert space defined by $S_{h}$ equipped with the norm of $L_{2}(\Omega)$. For instance, the derivative of this function with respect to $u$ applied to $w \in S_{h}$ is $P_{0}\left(\tilde{f}_{u}(\cdot, t, u) \cdot w\right) \in S_{h}$, which is clearly bounded in the $L_{2}$-norm, uniformly in $h$. Also, if $v_{h}=P_{0} v$ and $\|v\| \leqslant \rho$, we have $\left\|v_{h}\right\| \leqslant\|v\| \leqslant \rho$ and hence Theorem 1 implies that for a completely discrete solution obtained by discretization in time of (2.12) by a scheme of the above type, and with $v_{h}=P_{0} v$, we have

$$
\begin{aligned}
\left\|U_{n}-u\left(t_{n}\right)\right\| \leqslant C(\rho)\left\{\frac{h^{2}}{t_{n}} \log \frac{t_{n}}{h^{2}}+k\left[t_{n}^{-1} \log \frac{t_{n+1}}{k}+\left(\log \frac{t_{n+1}}{k}\right)^{2}\right]\right\} & \\
& \text { for } v \in L_{2}(\Omega) \text { with }\|v\| \leqslant \rho .
\end{aligned}
$$

We remark that in interesting applications of the type just described it is generally the case that $\tilde{f}$ and $\tilde{f}_{u}$ are unbounded for $u \in R$ so that, as $u$ is not necessarily bounded when $u \in L_{2}(\Omega)$, the above analysis does not apply. However, it is then often the case that by some independent argument, for instance by a maximum principle, the exact solution is known to be uniformly bounded in modulus by some constant $M$, say, in some interval $\bar{J}=[0, T]$, if the initial data are bounded, and that thus the values of $\tilde{f}(x, t, u)$ for $|u|>M$ do not influence the exact solution of (2.1). One can then modify $\tilde{f}$ for these values of $u$ in such a way that our assumptions become valid, thus changing the equation (2.1) without changing its solution for the initial data under consideration. With $F(k, t, u)$ based on the modified function, our assumptions (2.6) and (2.7) may remain valid. Note that this procedure might lead to a different discrete solution than the one based on the original $\tilde{f}$. Similarly, such a modification would change the semidiscrete equation (2.11) and thus also the totally discrete solution based on (2.12).

3. Runge-Kutta Methods. We recall (cf., e.g., [2] for details) that a Runge-Kutta method for the initial value problem

$$
\begin{aligned}
& y^{\prime}=g(t, y) \quad \text { for } t \geqslant 0, \\
& y(0)=y_{0},
\end{aligned}
$$

defines an approximate solution $Y_{n}$ at $t_{n}=n k$ successively by setting $Y_{0}=y_{0}$ and then determining $Y_{n+1}$ from $Y_{n}$ for $n \geqslant 0$ as follows: Let $t_{n j}=t_{n}+k \tau_{j}$ be given quadrature points with $\tau_{j} \geqslant 0$ for $j=1, \ldots, m$; define intermediate values $Y_{n j}$, $j=1, \ldots, m$, approximating $y\left(t_{n j}\right)$ by the nonlinear system

$$
Y_{n i}=Y_{n}+k \sum_{j=1}^{m} a_{i j} g\left(t_{n j}, Y_{n j}\right), \quad i=1, \ldots, m,
$$


and set finally

$$
Y_{n+1}=Y_{n}+k \sum_{j=1}^{m} b_{j} g\left(t_{n j}, Y_{n j}\right) .
$$

The coefficient matrix $\mathscr{A}=\left(a_{i j}\right)$ and the vector $b=\left(b_{1}, \ldots, b_{m}\right)^{\mathrm{T}}$ are associated with the quadrature formulae

$$
\int_{0}^{\tau_{i}} \Psi(t) d t \approx \sum_{j=1}^{m} a_{i j} \Psi\left(\tau_{j}\right)
$$

and

$$
\int_{0}^{1} \Psi(t) d t \approx \sum_{j=1}^{m} b_{j} \Psi\left(\tau_{j}\right),
$$

respectively, and we shall always assume that the latter is exact for constants, so that

$$
\sum_{j=1}^{m} b_{j}=1
$$

The method is implicit unless $\mathscr{A}$ is strictly lower triangular.

Applied to the parabolic problem (2.1), the method takes the form

$$
\begin{gathered}
U_{n i}=U_{n}+k \sum_{j=1}^{m} a_{i j}\left(-A U_{n j}+f\left(t_{n j}, U_{n j}\right)\right), \quad j=1, \ldots, m, \\
U_{n+1}=U_{n}+k \sum_{j=1}^{m} b_{j}\left(-A U_{n j}+f\left(t_{n j}, U_{n j}\right)\right) .
\end{gathered}
$$

We shall assume that $\mathscr{A}$ has no eigenvalues $\alpha_{j}$ with $\alpha_{j} \leqslant 0$ so that, in particular, the method is implicit and $I+z \mathscr{A}$ is nonsingular for $z \geqslant 0$. We set

$$
\begin{aligned}
& \sigma(z)=\left(\sigma_{i j}(z)\right)=(I+z \mathscr{A})^{-1}, \\
& s(z)=\left(s_{1}(z), \ldots, s_{m}(z)\right)^{\mathrm{T}}=\sigma(z) e \quad \text { where } e=(1, \ldots, 1)^{\mathrm{T}}, \\
& S(z)=\left(s_{i j}(z)\right)=\sigma(z) \mathscr{A}, \\
& r(z)=1-z b^{\mathrm{T}} s(z)=1-z b^{\mathrm{T}} \sigma(z) e, \\
& q(z)^{\mathrm{T}}=\left(q_{1}(z), \ldots, q_{m}(z)\right)=b^{\mathrm{T}} \sigma(z),
\end{aligned}
$$

and note that all these functions are bounded for $z \geqslant 0$, and that $\sigma, s, S$ and $q$ all vanish at $z=\infty$. With this notation, the equations (3.4) and (3.5) may be written

$$
\begin{aligned}
& U_{n i}=s_{i}(k A) U_{n}+k \sum_{j=1}^{m} s_{i j}(k A) f\left(t_{n j}, U_{n j}\right), \quad i=1, \ldots, m, \\
& U_{n+1}=r(k A) U_{n}+k \sum_{j=1}^{m} q_{j}(k A) f\left(t_{n j}, U_{n j}\right),
\end{aligned}
$$

where the rational functions of $k A$ are defined by spectral representation and thus, by the above, are all bounded linear operators on $H$.

Henceforth we shall restrict ourselves, as earlier in Section 2, to schemes such that $r(z)$ satisfies the strong stability property (1.3). Recall also that the method is accurate of order $p$, and, in particular, that (1.2) holds, if (3.1) and (3.2) are exact for 
polynomials of degree $p-2$ and $p-1$, respectively (cf. [2, p. 12]). For $p=1$ this reduces to the condition (3.3).

Since $f(t, \varphi)$ is Lipschitz continuous with respect to $\varphi$, it is easy to see that the nonlinear system (3.6) has a unique solution $\left(U_{n 1}, \ldots, U_{n m}\right)$ for $U_{n}$ given, and that it depends Lipschitz continuously on $U_{n}$. We may thus write our method in the form (2.4), where

$$
F(k, t, \varphi)=\sum_{j=1}^{m} q_{j}(k A) f\left(t+\tau_{j} k, \varphi_{j}\right),
$$

with $\varphi_{j}=\varphi_{j}(k, t, \varphi)$ defined by

$$
\varphi_{i}=s_{i}(k A) \varphi+k \sum_{j=1}^{m} q_{j}(k A) f\left(t+\tau_{j} k, \varphi_{j}\right), \quad i=1, \ldots, m .
$$

We show that this method satisfies the conditions of Theorem 1. In fact, (2.6) is obvious and it remains only to consider (2.7). We write

$$
\begin{aligned}
F(k, t, \varphi)-f(t, \varphi)= & \sum_{j=1}^{m} q_{j}(k A)\left(f\left(t+\tau_{j} k, \varphi_{j}\right)-f\left(t+\tau_{j} k, \varphi\right)\right) \\
& +\sum_{j=1}^{m} q_{j}(k A)\left(f\left(t+\tau_{j} k, \varphi\right)-f(t, \varphi)\right) \\
& +\left(\sum_{j=1}^{m} q_{j}(k A)-I\right) f(t, \varphi) \\
= & \delta_{1}+\delta_{2}+\delta_{3} .
\end{aligned}
$$

Here

$$
\left\|A^{-1} \delta_{1}\right\| \leqslant C\left\|\delta_{1}\right\| \leqslant C \sum_{j=1}^{m}\left\|\varphi_{j}-\varphi\right\|
$$

and, noting that $s_{i}(0)=1$, we have easily from (3.7)

$$
\left\|\varphi_{i}-\varphi\right\| \leqslant\left\|\left(s_{i}(k A)-I\right) \varphi\right\|+C k \leqslant C k(\|A \varphi\|+1) .
$$

Further, it is obvious that

$$
\left\|A^{-1} \delta_{2}\right\| \leqslant C\left\|\delta_{2}\right\| \leqslant C k
$$

Finally, since by (3.3)

$$
\sum_{j=1}^{m} q_{j}(0)=\sum_{j=1}^{m} b_{j}=1
$$

we obtain

$$
\left\|A^{-1} \delta_{3}\right\|=k\left\|(k A)^{-1}\left(\sum_{j=1}^{m} q_{j}(k A)-I\right) f\right\| \leqslant C k .
$$

Together, these estimates complete the proof that the Runge-Kutta methods under consideration satisfy the assumptions of Theorem 1 . 
4. The counterexample. We shall now show that, at least for methods of RungeKutta type, the estimate of Theorem 1 is essentially best possible. For this purpose we introduce the unidimensional parabolic system

$$
\begin{aligned}
& u_{t}-u_{x x}=v^{2} \\
& v_{t}-v_{x x}=0
\end{aligned} \quad \text { in }[0, \pi] \times J,
$$

with the boundary and initial conditions

$$
\begin{aligned}
& u(0, t)=u(\pi, t)=v(0, t)=v(\pi, t)=0, \\
& u(x, 0)=0, \quad v(x, 0)=w(x),
\end{aligned}
$$

which we consider in the Hilbert space $H=L_{2}(0, \pi)^{2}$, with the obvious corresponding definition of $A$. We note that by the maximum principle $\|v(t)\|_{L_{\infty}(\Omega)}$ is nonincreasing and hence $u(t)$ is uniformly bounded on $J$ for all $w$ with a common uniform bound.

We consider now the discrete solution $\left(U_{n}, V_{n}\right)$ of (4.1), (4.2), defined by a Runge-Kutta method as described above. We shall show that for no $t \in J$ is it possible to find $C=C(t)$ such that the error, measured in the norm in $L_{2}(0, \pi)^{2}$, is bounded by $C k \varepsilon_{k}$ with $\varepsilon_{k} \rightarrow 0$ as $k \rightarrow 0$, for all $w$ with $\|w\|_{L_{\infty}} \leqslant 1$, say. More precisely, we shall show that there is a positive constant $c$ such that for initial data of the form $w=\sin N x$ with $N=1,2, \ldots$ it is possible to find associated sequences $\left\{n_{N}\right\}$ and $\left\{k_{N}\right\}$ with $n_{N} k_{N}=t$ such that the error in the $u$-component satisfies

$$
\left\|U_{n_{N}}-u(t)\right\|_{L_{2}(0, \pi)} \geqslant c k_{N} \text { for large } N \text {. }
$$

This will be demonstrated by showing that the corresponding estimate holds for the first Fourier coefficient of the error.

For the $v$-component of the exact solution of (4.1), (4.2) we have at once

$$
v(t)=e^{-N^{2} t} \sin N x
$$

which gives for the determination of $u$ the inhomogeneous linear equation

$$
\begin{aligned}
& u_{t}-u_{x x}=e^{-2 N^{2} t} \sin ^{2} N x \quad \text { for } t \in J, \\
& u(0)=0 .
\end{aligned}
$$

Similarly, we have for the corresponding discrete problem, using the Runge-Kutta method, with our above notation,

$$
V_{n}=r\left(k N^{2}\right)^{n} \sin N x \text { for } t_{n} \in \bar{J},
$$

and thus also

$$
V_{n i}=s_{i}\left(k N^{2}\right) r\left(k N^{2}\right)^{n} \sin N x, \quad i=1, \ldots, m,
$$

and hence for the determination of $U_{n}$ the recursion formula

$$
\begin{aligned}
& U_{n+1}=r(k A) U_{n}+k \sum_{j=1}^{m} q_{j}(k A) s_{j}\left(k N^{2}\right)^{2} r\left(k N^{2}\right)^{2 n} \sin ^{2} N x, \\
& U_{0}=0 .
\end{aligned}
$$

We now introduce the first Fourier coefficients of $u(t)$ and $U_{n}$,

$$
\alpha(t)=\frac{2}{\pi} \int_{0}^{\pi} u(x, t) \sin x d x \quad \text { and } \quad \bar{\alpha}_{n}=\frac{2}{\pi} \int_{0}^{\pi} U_{n}(x) \sin x d x
$$


We obtain from (4.4)

$$
\begin{aligned}
& \alpha^{\prime}+\alpha=\frac{2}{\pi} e^{-2 N^{2} t} \int_{0}^{\pi} \sin x \sin ^{2} N x d x=\frac{8 N^{2}}{\pi\left(4 N^{2}-1\right)} e^{-2 N^{2} t}, \\
& \alpha(0)=0,
\end{aligned}
$$

which yields

$$
\alpha(t)=\frac{8 N^{2}}{\pi\left(4 N^{2}-1\right)} \frac{1}{2 N^{2}-1}\left(e^{-t}-e^{-2 N^{2} t}\right) .
$$

Correspondingly, from (4.5),

$$
\begin{aligned}
& \bar{\alpha}_{n+1}=r(k) \bar{\alpha}_{n}+k \sum_{j=1}^{m} q_{j}(k) s_{j}\left(k N^{2}\right)^{2} r\left(k N^{2}\right)^{2 n} \int_{0}^{\pi} \sin x \sin ^{2} N x d x, \\
& \bar{\alpha}_{0}=0
\end{aligned}
$$

and hence

$$
\begin{aligned}
\bar{\alpha}_{n} & =\frac{8 N^{2}}{\pi\left(4 N^{2}-1\right)} \sum_{i=0}^{n-1} r(k)^{n-1-i} k \sum_{j=1}^{m} q_{j}(k) s_{j}\left(k N^{2}\right)^{2} r\left(k N^{2}\right)^{2 i} \\
& =\frac{8 N^{2}}{\pi\left(4 N^{2}-1\right)} k \sum_{j=1}^{m} q_{j}(k) s_{j}\left(k N^{2}\right)^{2} \frac{r(k)^{n}-r\left(k N^{2}\right)^{2 n}}{r(k)-r\left(k N^{2}\right)^{2}}
\end{aligned}
$$

We write

$$
\begin{aligned}
\frac{\bar{\alpha}_{n}-\alpha(t)}{k}=\frac{8 N^{2}}{\pi\left(4 N^{2}-1\right)}\left\{\sum_{j=1}^{m} q_{j}(k) s_{j}\left(k N^{2}\right)^{2} \frac{r(k)^{n}-r\left(k N^{2}\right)^{2 n}}{r(k)-r\left(k N^{2}\right)^{2}}\right. \\
\left.-\frac{1}{2 N^{2}-1} \frac{1}{k}\left(e^{-t}-e^{-2 N^{2} t}\right)\right\} .
\end{aligned}
$$

We now fix $t$ positive and set, with $M$ a fixed positive integer, $n_{N}=M N^{2}$ and $k_{N}=t / n_{N}$ so that $k_{N} N^{2}=t / M=t_{0}$. We note that since $r(z)=e^{-z}+O\left(z^{2}\right)$ for small $z$, we have $r(0)=1$ and $r\left(k_{N}\right)^{n_{N}}=r\left(t / n_{N}\right)^{n_{N}} \rightarrow e^{-t}$ as $N \rightarrow \infty$. Further, since $\left|r\left(t_{0}\right)\right|<1$, we have $r\left(k_{N} N^{2}\right)^{2 n_{N}}=r\left(t_{0}\right)^{2 n_{N}} \rightarrow 0$ as $N \rightarrow \infty$, and since $q_{j}(0)$ $=b_{j}$, we conclude

$$
\lim _{N \rightarrow \infty} \frac{\bar{\alpha}_{n_{N}}-\alpha(t)}{k_{N}}=\frac{2}{\pi} e^{-t}\left\{\sum_{j=1}^{m} b_{j} s_{j}\left(t_{0}\right)^{2} \frac{1}{1-r\left(t_{0}\right)^{2}}-\frac{1}{2 t_{0}}\right\} .
$$

We shall show that for $M$ suitably. chosen the last factor is nonzero, which then shows that, for large $N$,

$$
\left\|U_{n_{N}}-u(t)\right\|_{L_{2}(0, \pi)} \geqslant\left(\frac{\pi}{2}\right)^{1 / 2}\left|\bar{\alpha}_{n_{N}}-\alpha(t)\right| \geqslant c k_{N} \quad \text { with } c=c(t)>0,
$$

and thus completes the proof of (4.3). Assume therefore that for any choice of $M$ the expression inside the parentheses in (4.6) vanishes. In such a case, this rational function vanishes identically, and we have

$$
2 z \sum_{j=1}^{m} b_{j} s_{j}(z)^{2} \equiv 1-r(z)^{2}
$$


But for large $z, s_{j}(z)=O\left(z^{-1}\right)$ so that, by letting $z$ tend to infinity, we obtain $|r(\infty)|=1$, contrary to our hypothesis. This completes the proof.

We note that the right-hand side of (4.1) is not bounded for $v \in R$ so that, formally, the assumptions of Section 2 are not satisfied for (4.1), (4.2). However, both $|v(t)|$ and $\left|V_{n}\right|$ are bounded by 1 and $\left|V_{n i}\right|$ by $K=\max _{i} \sup _{z} \geqslant 0\left|s_{i}(z)\right|$. Hence only the values of the right-hand side for $|v| \leqslant K$ enter the calculations, and we may replace $v^{2}$ by a smooth function $f(v)$ which satisfies our previous conditions and agrees with $v^{2}$ for $|v| \leqslant K$, without changing either the exact or the approximate solutions.

5. A Higher-Order Result for a Class of Runge-Kutta Methods. Although for initial data in $H$ it was only possible, above, to show an essentially first-order error estimate, it may still be possible to do better for initial data which are more regular, but not regular enough for optimal order estimates to hold uniformly down to $t=0$. In this section we shall show a $O\left(k^{p}\right)$ error estimate for a Runge-Kutta type method based on quadrature formulas of orders $p-1$ for the intermediate points and $p$ for the whole interval, and for the case that $u^{(p)}$ and $f(t, u)^{(p)}$ are of order $O\left(t^{-1}\right)$ for small $t$.

THEOREM 2. Let $U_{n}$ be the discrete solution of (2.1) by a Runge-Kutta scheme satisfying (1.3) and for which the quadrature values (3.1) and (3.2) are exact for all polynomials of degree $p-2$ and $p-1$, respectively. Then there is a constant $C=C(\rho)$ such that

$$
\left\|U_{n}-u\left(t_{n}\right)\right\| \leqslant C k^{p}\left(t_{n}^{-1} \log \frac{t_{n+1}}{k}+\left(\log \frac{t_{n+1}}{k}\right)^{2}\right) \quad \text { for } t_{n} \in J
$$

if, with $\varphi(t)=f(t, u(t))$,

$$
\max \left(\max _{j \leqslant p-1}\left(\left\|u^{(j)}\right\|,\left\|\varphi^{(j)}\right\|\right), t\left\|u^{(p)}\right\|, t\left\|\varphi^{(p)}\right\|\right) \leqslant \rho .
$$

The constant $C$ is independent of the particular choice of the Hilbert space $H$ and the positive definite operator $A$.

Proof. Let us introduce the error functional for the quadrature formulae (3.1) and (3.2), transformed to the interval $I_{n}$, i.e.,

$$
\begin{aligned}
& Q_{n, j}(\Psi)=\int_{t_{n}}^{t_{n j}} \Psi d s-k \sum_{l=1}^{m} a_{j l} \Psi\left(t_{n l}\right), \quad j=1, \ldots, m, \\
& Q_{n}(\Psi)=\int_{t_{n}}^{t_{n+1}} \Psi d s-k \sum_{l=1}^{m} b_{l} \Psi\left(t_{n l}\right) .
\end{aligned}
$$

Recall that our assumptions that (3.1) and (3.2) are exact for polynomials of degree $p-1$ and $p-2$, respectively, imply

$$
\begin{gathered}
\left\|Q_{n j}(\Psi)\right\| \leqslant C k^{l+1} \sup _{I_{n}}\left\|\Psi^{(l)}\right\| \text { for } l \leqslant p-1, \\
\left\|Q_{n}(\Psi)\right\| \leqslant C k^{l+1} \sup _{I_{n}}\left\|\Psi^{(l)}\right\| \text { for } l \leqslant p .
\end{gathered}
$$


We note now that $Q_{n j}\left(u_{t}\right)$ and $Q_{n}\left(u_{t}\right)$ are the truncation errors in (3.4) and (3.5), or that

$$
\begin{gathered}
u\left(t_{n i}\right)=u\left(t_{n}\right)+k \sum_{j=1}^{m} a_{i j}\left(-A u\left(t_{n j}\right)+f\left(t_{n j}, u\left(t_{n j}\right)\right)\right)+Q_{n i}\left(u_{t}\right), \\
\quad i=1, \ldots, m, \\
u\left(t_{n+1}\right)=u\left(t_{n}\right)+k \sum_{j=1}^{m} b_{j}\left(-A u\left(t_{n j}\right)+f\left(t_{n j}, u\left(t_{n j}\right)\right)\right)+Q_{n}\left(u_{t}\right) .
\end{gathered}
$$

It follows by obvious calculations that

$$
\begin{aligned}
u\left(t_{n i}\right)= & s_{i}(k A) u\left(t_{n}\right)+k \sum_{j=1}^{m} s_{i j}(k A) f\left(t_{n j}, u\left(t_{n j}\right)\right) \\
& +\sum_{j=1}^{m} \sigma_{i j}(k A) Q_{n j}\left(u_{t}\right), \quad i=1, \ldots, m, \\
u\left(t_{n+1}\right)= & r(k A) u\left(t_{n}\right)+k \sum_{j=1}^{m} q_{j}(k A) f\left(t_{n j}, u\left(t_{n j}\right)\right) \\
& -\sum_{j=1}^{m} k A q_{j}(k A) Q_{n j}\left(u_{t}\right)+Q_{n}\left(u_{t}\right) .
\end{aligned}
$$

Hence for the errors $e_{n}=U_{n}-u\left(t_{n}\right), e_{n j}=U_{n j}-u\left(t_{n j}\right)$, we have

$$
\begin{aligned}
e_{n i}= & s_{i}(k A) e_{n}+k \sum_{j=1}^{m} s_{i j}(k A)\left(f\left(t_{n j}, U_{n j}\right)-f\left(t_{n j}, u\left(t_{n j}\right)\right)\right) \\
& -\sum_{j=1}^{m} \sigma_{i j}(k A) Q_{n j}\left(u_{t}\right), \\
e_{n+1}= & r(k A) e_{n}+k \sum_{j=1}^{m} q_{j}(k A)\left(f\left(t_{n, j}, U_{n, j}\right)-f\left(t_{n j}, u\left(t_{n j}\right)\right)\right) \\
& +\sum_{j=1}^{m} k A q_{j}(k A) Q_{n j}\left(u_{t}\right)-Q_{n}\left(u_{t}\right) \\
= & r(k A) e_{n}+\eta_{n 1}+\eta_{n 2}+\eta_{n 3},
\end{aligned}
$$

and, where as in Section 2 we have set $E_{k}=r(k A)$,

$$
e_{n}=\sum_{j=0}^{n-1} E_{k}^{n-1-j}\left(\eta_{j 1}+\eta_{j 2}+\eta_{j 3}\right) .
$$

We obtain at once from (5.4), for $k$ small,

$$
\sum_{i=1}^{m}\left\|e_{n i}\right\| \leqslant C\left\|e_{n}\right\|+C \sum_{j=1}^{m}\left\|Q_{n j}\left(u_{t}\right)\right\|
$$

and hence, using alsó (5.2), for $j=1, \ldots, n-1$,

$$
\begin{aligned}
\left\|\eta_{j 1}\right\| & \leqslant C k \sum_{l=1}^{m}\left\|e_{j l}\right\| \leqslant C k\left(\left\|e_{j}\right\|+\sum_{l=1}^{m}\left\|Q_{j l}\left(u_{t}\right)\right\|\right) \\
& \leqslant C k\left\|e_{j}\right\|+C k^{p+1} \sup _{I_{j}}\left\|u^{(p)}\right\| \leqslant C k\left\|e_{j}\right\|+C k^{p+1} t_{j}^{-1} \\
& \leqslant C k\left\|e_{j}\right\|+C k^{p+1} t_{j+1}^{-1},
\end{aligned}
$$


and, for $j=0$,

$$
\left\|\eta_{01}\right\| \leqslant C k\left\|e_{0}\right\|+C k^{p} \sup _{I_{j}}\left\|u^{(p-1)}\right\| \leqslant C k\left\|e_{0}\right\|+C k^{p+1} t_{1}^{-1}
$$

so that for the sum in (5.5) with $\eta_{j 1}$,

$$
\begin{aligned}
\left\|\sum_{j=0}^{n-1} E_{k}^{n-1-j} \eta_{j 1}\right\| & \leqslant C k \sum_{j=0}^{n-1}\left\|e_{j}\right\|+C k^{p+1} \sum_{j=0}^{n-1} \frac{1}{t_{j+1}} \\
& \leqslant C k \sum_{j=0}^{n-1}\left\|e_{j}\right\|+C k^{p} \log \frac{t_{n+1}}{k} .
\end{aligned}
$$

In order to estimate the term involving $\eta_{j 2}$, we note that since $z q(z)$ is bounded, we have, using the property (1.3),

$$
\left|r(z)^{n} z q(z)\right| \leqslant \frac{C}{n+1}=C \frac{k}{t_{n+1}}
$$

and hence, for $j=1, \ldots, n-1$,

$$
\begin{aligned}
\left\|E_{k}^{n-1-j} \eta_{j 2}\right\| & \leqslant C \frac{k}{t_{n-j}} \sum_{l=1}^{m}\left\|Q_{j l}\left(u_{t}\right)\right\| \leqslant C \frac{k^{p+1}}{t_{n-j}} \sup _{I_{j}}\left\|u^{(p)}\right\| \\
& \leqslant C k^{p+1} \frac{1}{t_{n-j} t_{j}}=C \frac{k^{p+1}}{t_{n}}\left(\frac{1}{t_{j}}+\frac{1}{t_{n-j}}\right),
\end{aligned}
$$

so that, again with an obvious modification for the term with $j=0$,

$$
\left\|\sum_{j=0}^{n-1} E_{k}^{n-1-j} \eta_{j 2}\right\| \leqslant C \frac{k^{p+1}}{t_{n}} \sum_{j=0}^{n-1}\left(\frac{1}{t_{j+1}}+\frac{1}{t_{n-j}}\right) \leqslant C \frac{k^{p}}{t_{n}} \log \frac{t_{n+1}}{k} .
$$

It remains to estimate the term in $\eta_{j 3}$ which we write

$$
\eta_{j 3}=Q_{j}\left(u_{t}\right)=Q_{j}(-A u+\varphi(u))=-A Q_{j}(u)+Q_{j}(\varphi) \text {. }
$$

For the second term we have by (5.3) and our assumption (5.1)

$$
\left\|Q_{j}(\varphi)\right\| \leqslant C \frac{k^{p+1}}{t_{j+1}}=C \frac{k^{p}}{j+1} \quad \text { for } j=0, \ldots, n-1,
$$

and hence

$$
\left\|\sum_{j=0}^{n-1} E_{k}^{n-1-j} Q_{j}(\varphi)\right\| \leqslant C k^{p} \log \frac{t_{n+1}}{k} .
$$

To estimate the first, we note that

$$
\begin{aligned}
\left\|E_{k}^{n} A v\right\| & \leqslant\left\|\left(r(k A)^{n}-e^{-n k A}\right) A v\right\|+\left\|e^{-n k A} A v\right\| \\
& \leqslant C \frac{k}{t_{n+1}}\|A v\|+\frac{C}{t_{n+1}}\|v\| \leqslant \frac{C}{t_{n+1}}(\|v\|+k\|A v\|) \text { for } n \geqslant 0
\end{aligned}
$$

(if $r(\infty)=0$ we have more directly $\left\|E_{k}^{n} A v\right\|=\left\|r(k A)^{n} A v\right\| \leqslant C t_{n}^{-1}\|v\|$ ), and hence, using (5.3) with $l=p$ and $l=p-1$, and noting also that $t A u^{(p-1)}$ is bounded by our assumptions,

$$
\left\|E_{k}^{n-1-j} A Q_{j}(u)\right\| \leqslant \frac{C}{t_{n-j}}\left(\left\|Q_{j}(u)\right\|+k\left\|Q_{j}(A u)\right\|\right) \leqslant C k^{p+1} \frac{1}{t_{n-j} t_{j+1}},
$$


so that

$$
\sum_{j=0}^{n-1}\left\|E_{k}^{n-1-j} A Q_{j}(u)\right\| \leqslant C \frac{k^{p}}{t_{n}} \log \frac{t_{n+1}}{k} .
$$

Altogether, we thus have

$$
\left\|e_{n}\right\| \leqslant C k \sum_{j=0}^{n-1}\left\|e_{j}\right\|+C k^{p} t_{n}^{-1} \log \frac{t_{n+1}}{k},
$$

from which our result follows in exactly the same way as in the proof of Theorem 1.

As an example where Theorem 2 applies, consider as in Section 2 the case that $H=L_{2}(\Omega)$, where $\Omega$ is a domain with smooth boundary in $R^{d}$, now with $d \leqslant 3$, where $A=-\Delta$ with $D(A)=H_{0}^{1}(\Omega) \cap H^{2}(\Omega)$, and where $f(t, u)$ is generated by a smooth function $\tilde{f}(x, t, u)$ on $\bar{\Omega} \times \bar{J} \times R$ which is bounded together with its derivatives of first and second order in $t$ and $u$. The equation (2.1) thus reads

$$
\begin{array}{ll}
u_{t}-\Delta u=\tilde{f}(x, t, u) & \text { in } \Omega \times J, \\
u=0 & \text { on } \partial \Omega \times J, \\
u(x, 0)=v(x) & \text { in } \Omega .
\end{array}
$$

Assuming now that $v \in D(A)$ with $\|\Delta v\|=\|\Delta v\|_{L_{2}(\Omega)} \leqslant \rho$, it follows from Lemma 1 of Section 2 that $u$ and $u^{\prime}=u_{t}$ are bounded in $H$ for $t \in \bar{J}$. To see that also $t u^{\prime \prime}=t u_{t t}$ is bounded in $H$, we differentiate (5.6) twice to obtain

$$
u_{t t}-\Delta u_{t}=\tilde{f}_{t}+\tilde{f}_{u} \cdot u_{t}
$$

and

$$
u_{t t t}-\Delta u_{t t}=\tilde{f}_{t t}+2 \tilde{f}_{t u} u_{t}+\tilde{f}_{u u} \cdot u_{t}^{2}+\tilde{f}_{u} u_{t t}
$$

Note that, since $d \leqslant 3$, we have

$$
\|\varphi\|_{L_{\infty}(\Omega)} \leqslant C\|\varphi\|_{H^{2}(\Omega)} \leqslant C\|\Delta \varphi\| \text { for } \varphi \in D(A) .
$$

Therefore, since $u_{t}$ is bounded in $H$, we obtain from (5.7)

$$
\left\|u_{t}^{2}\right\| \leqslant\left\|u_{t}\right\|_{L_{\infty}(\Omega)}\left\|u_{t}\right\| \leqslant C\left\|\Delta u_{t}\right\| \leqslant C\left\|u_{t t}\right\|+C .
$$

Multiplication of (5.8) by $t^{2} u_{t t}$ and integration over $\Omega$ gives

$$
\begin{aligned}
\frac{1}{2} \frac{d}{d t}\left(t^{2}\left\|u_{t t}\right\|^{2}\right)+t^{2}\left\|\nabla u_{t t}\right\|^{2} & \leqslant C t^{2}\left(\left\|u_{t t}\right\|^{2}+\left\|u_{t t}\right\|\right)+t\left\|u_{t t}\right\|^{2} \\
& \leqslant C t\left\|u_{t t}\right\|^{2}+C,
\end{aligned}
$$

and hence

$$
t^{2}\left\|u_{t t}\right\|^{2} \leqslant C \int_{0}^{t} s\left\|u_{t t}\right\|^{2} d s+C .
$$

Multiplication of (5.7) by $t u_{t t}$ and integrating gives similarly

$$
\int_{0}^{t} s\left\|u_{t t}\right\|^{2} d s \leqslant C \int_{0}^{t}\left\|\nabla u_{t}\right\|^{2} d s+C,
$$

and using instead (5.7) multiplied by $u_{t}$ we have finally

$$
\int_{0}^{t}\left\|\nabla u_{t}\right\|^{2} d s \leqslant C\left\|u_{t}(0)\right\|^{2}+C \leqslant C\|\Delta v\|^{2}+C \leqslant C .
$$


Together, our estimates show the boundedness of $t u_{t t}$ in $H$. We also have for $\varphi(t)=f(t, u(t))$ that $\varphi$ and $\varphi^{\prime}=\tilde{f}_{t}+\tilde{f}_{u} u$, are bounded. Finally, in order to see that $t \varphi^{\prime \prime}$ is bounded, we note that by the above,

$$
\left\|\varphi^{\prime \prime}\right\|=\left\|\tilde{f}_{t t}+2 \tilde{f}_{u t} u_{t}+\tilde{f}_{u u} u_{t}^{2}+\tilde{f}_{u} u_{t t}\right\| \leqslant C\left(\left\|u_{t t}\right\|+1\right) \leqslant C t^{-1} .
$$

The assumptions of Theorem 2 are thus satisfied with $p=2$, and we conclude that for such methods

$$
\left\|U_{n}-u\left(t_{n}\right)\right\| \leqslant C(\rho) k^{2}\left(t_{n}^{-1} \log \frac{t_{n+1}}{k}+\left(\log \frac{t_{n+1}}{k}\right)^{2}\right) \quad \text { if }\|\Delta v\| \leqslant \rho .
$$

The same method can be applied to the discretization in time of the equation obtained from (5.6) by discretization in space. Consider for example, as in Section 2, the semidiscrete equation (2.11) or (2.12) with continuous piecewise linear approximating functions, now on a quasiuniform partitioning of $\Omega$. With $v_{h}=P_{1} v=-T_{h} \Delta v$ the elliptic projection of $v$ onto $S_{h}$, we have for $v \in D(\Delta)$

$$
\left\|u_{h, t}(0)\right\|=\left\|\Delta_{h} v_{h}+P_{0} f\left(0, v_{h}\right)\right\| \leqslant\|\Delta v\|+C .
$$

It is easy to show that the error in the semidiscrete solution is then bounded as

$$
\left\|u_{h}(t)-u(t)\right\| \leqslant C(\rho) h^{2} \text { for } \Delta v \leqslant \rho,
$$

and we also conclude by Lemma 1 that $u_{h}$ and $u_{h, t}$ are bounded in $L_{2}(\Omega)$ with $\Delta v$. The same arguments as above will then show that $t u_{h, t t}$ is bounded in $L_{2}(\Omega)$, uniformly in $h$, provided only that the analogue of (5.9) is valid in the present situation, namely

$$
\|\chi\|_{L_{\infty}(\Omega)} \leqslant C\left\|\Delta_{h} \chi\right\| \quad \text { for } \chi \in S_{h},
$$

or, equivalently,

$$
\left\|T_{h} \chi\right\|_{L_{\infty}\left(\Omega_{h}\right)} \leqslant C\|\chi\| \text { for } \chi \in S_{h},
$$

where $\Omega_{h}(\subset \Omega)$ is the union of the simplices in the definition of $S_{h}$. But with $T=(-\Delta)^{-1}$ we have by $(5.9)$

$$
\|T \chi\|_{L_{\infty}(\Omega)} \leqslant C\|\chi\|,
$$

and it remains to estimate $\left(T_{h}-T\right) \chi$, the error in the elliptic problem with right-hand side $\chi$. By well-known error and regularity estimates for the elliptic problem (cf. Schatz and Wahlbin [4]) and an inverse estimate to estimate the norm of $\chi$ in $H^{1 / 2+\varepsilon}(\Omega)$ by that in $L_{2}(\Omega)$, we have with $0<\varepsilon<\frac{1}{2}$,

$$
\begin{aligned}
\left\|\left(T_{h}-T\right) \chi\right\|_{L_{\infty}\left(\Omega_{h}\right)} & \leqslant C \log \frac{1}{h} \inf _{\tilde{\chi} \in S_{h}}\|T \chi-\tilde{\chi}\|_{L_{\infty}\left(\Omega_{h}\right)} \leqslant C h \log \frac{1}{h}\|T \chi\|_{W_{\infty}^{1}(\Omega)} \\
& \leqslant C h \log \frac{1}{h}\|T \chi\|_{H^{5 / 2+\varepsilon}(\Omega)} \leqslant C h \log \frac{1}{h}\|\chi\|_{H^{1 / 2+\varepsilon}(\Omega)} \\
& \leqslant C h^{1 / 2-\varepsilon} \log \frac{1}{h}\|\chi\| \leqslant C\|\chi\| .
\end{aligned}
$$

Together, our estimates show (5.10) and thus complete the proof that $t u_{h, t t}$ is bounded. As above, it follows that $\varphi_{h}=P_{0} \tilde{f}(\cdot, t, u)$ is bounded together with $\varphi_{h}^{\prime}$ and $t \varphi_{h}^{\prime \prime}$, uniformly in $h$, and we conclude by Theorem 2 for the completely discrete 
solution $U_{n}$ obtained by discretization in time of (2.12) that

$$
\begin{aligned}
& \left\|U_{n}-u\left(t_{n}\right)\right\| \leqslant C(\rho)\left\{h^{2}+k^{2}\left[t_{n}^{-1} \log \frac{t_{n+1}}{k}+\left(\log \frac{t_{n+1}}{k}\right)^{2}\right]\right\} \\
& \text { for } v \in D(A) \text { with }\|\Delta v\| \leqslant \rho .
\end{aligned}
$$

For methods which are higher-order in space, a higher power of $h$ may be obtained in combination with some negative power of $t_{n}$.

We close by exhibiting two examples of methods which satisfy our assumptions with $p=2$ (cf. [2]). First let

$$
\tau_{1}=\frac{1}{3}, \quad \tau_{2}=1, \quad \mathscr{A}=\left(\begin{array}{cc}
\frac{5}{12} & -\frac{1}{12} \\
\frac{3}{4} & \frac{1}{4}
\end{array}\right), \quad b^{\mathrm{T}}=\left(\frac{3}{4}, \frac{1}{4}\right) .
$$

Then $U_{n+1}=U_{n 2}$ and $r(z)=\left(1-\frac{1}{3} z\right) /\left(1+\frac{2}{3} z+\frac{1}{6} z^{2}\right)$ is the Padé approximant of $e^{-z}$ of orders $(1,2)$ and satisfies $r(\infty)=0$. Secondly, with

$$
\tau_{1}=\frac{1}{2}+\frac{1}{2 \sqrt{3}}, \quad \tau_{2}=\frac{1}{2}-\frac{1}{2 \sqrt{3}}, \quad \mathscr{A}=\left(\begin{array}{rr}
\tau_{1} & 0 \\
-\frac{1}{\sqrt{3}}, & \tau_{1}
\end{array}\right), \quad b^{\mathrm{T}}=\left(\frac{1}{2}, \frac{1}{2}\right),
$$

we have (cf. Calahan's scheme)

$$
r(z)=1-\frac{z}{1+\tau_{1} z}-\frac{\sqrt{3}}{6}\left(\frac{z}{1+\tau_{1} z}\right)^{2} \text { with }|r(\infty)|<1 .
$$

IRISA

UER Mathématiques et Informatique

Université de Rennes I

Campus de Beaulieu

35042 Rennes Cédex, France

Department of Mathematics

Chalmers University of Technology

S-412 96 Göteborg, Sweden

1. G. A. Baker, J. H. Bramble \& V. ThomeE, "Single step Galerkin approximations for parabolic problems," Math. Comp., v. 31, 1977, pp. 818-847.

2. M. Crouzerx, Sur l'Approximation des Équations Différentielles Opérationnelles Linéaires par des Méthodes de Runge-Kutta, Thèse, Université Paris VI, 1975.

3. C. Johnson, S. Larsson, V. Thomke \& L. B. Wahlbin, "Error estimates for spatially discrete approximations of semilinear parabolic equations with nonsmooth initial data," Math. Comp., v. 49, 1987, pp. 331-357.

4. A. H. SChatz \& L. B. WAHLBIN, "On the quasi-optimality in $L_{\infty}$ of the $\stackrel{H}{H}^{1}$-projection into finite element spaces," Math. Comp. v. 38, 1982, pp. 1-22. 